\title{
Shape-Based Analysis on Component-Graphs for Multivalued Image Processing ${ }^{\star}$
}

\author{
Éloïse Grossiord ${ }^{1,4}$, Benoît Naegel ${ }^{2}$, Hugues Talbot ${ }^{1}$, Nicolas Passat $^{3}$, \\ Laurent Najman ${ }^{1}$ \\ ${ }^{1}$ Université Paris-Est, ESIEE-Paris, LIGM, CNRS, France \\ ${ }^{2}$ Université de Strasbourg, ICube, CNRS, France \\ ${ }^{3}$ Université de Reims Champagne-Ardenne, CReSTIC, France \\ ${ }^{4}$ KeoSys, Nantes, France
}

\begin{abstract}
The extension of mathematical morphology to multivalued images is an important issue. This is particularly true in the context of connected operators based on morphological hierarchies, which aim to provide efficient image filtering and segmentation tools in various application fields, e.g.(bio)medical imaging, remote sensing, or astronomy. In this article, we propose a preliminary study that describes how two notions recently introduced for connected filtering, namely component-graphs (that extend component-trees from a spectral point of view) and shaping (that extend component-trees from a conceptual point of view) can be associated for the effective processing of multivalued images. Structural, algorithmic and experimental developments are proposed. This study opens the way to new paradigms for connected filtering based on hierarchies.
\end{abstract}

Keywords: Connected filtering, morphological hierarchies, component-graph, component-tree, shaping, multivalued images, medical imaging.

\section{Introduction}

Connected operators have been intensively studied for the last twenty years in the framework of mathematical morphology [1]. In this context, operators based on hierarchical image models (i.e., trees) have been the object of several structural, algorithmic and methodological developments [2], in order to tackle specific issues associated to various application fields.

In the meantime, mathematical morphology - first defined on binary, and then on grey-level images [3] - progressively extended its framework and tools to the case of multivalued images [4], with a strong focus on colour imaging, but also with contributions in label, multimodal, multi- and hyperspectral imaging.

At the convergence of both issues, a question naturally arises: How can we perform connected filtering on multivalued images based on morphological hierarchies? Two principal answers were given to this question. The first defined morphological trees by

\footnotetext{
* This research was partially funded by the French Agence Nationale de la Recherche (Grant Agreement ANR-10-BLAN-0205) and the Programme d'Investissements d'Avenir (LabEx Bézout, ANR-10-LABX-58).
} 
considering a simplifying metric (e.g., a saliency measure for hierarchical watersheds [5], a merging order for partition trees [6,7], or via hyperconnections [8]) in their construction process. The second simplified the multivalued space of images a priori, to retrieve tractable totally ordered values, e.g., by marginal or vectorial policies [4].

The latter strategy allows us to rely on morphological trees specifically designed for grey-level images, namely component-trees [9] and trees of shapes [10]. The simplification of multivalued space however induces a loss of information. To cope with this problem, efforts were conducted to extend these data-structures to such complex spaces. Nevertheless, preserving a tree structure still requires a final simplification [11], or restrictive constraints on the value space [12]. Indeed, a true extension of such hierarchies to multivalued spaces necessarily leads to a data-structure that is no longer a tree, but a directed acyclic graph. This is in particular the case for the notion of component-graph [13], that extends the component-tree.

The higher richness and structural complexity of the component-graph, with respect to the component-tree, induces algorithmic issues when considering the classical antiextensive filtering process developed in $[9,14]$. This is in particular the case for handling the spatial complexity [15], pruning policies and image reconstruction [16].

Recently, a new notion of shaping [17] was introduced as an efficient way to improve the framework of anti-extensive filtering of $[9,14]$, by considering a two-layer component-tree for grey-level image processing $[18,19]$.

The key-idea of this article is to consider that the paradigm of shaping can be used not only to build a tree on a tree, but also a tree on a graph. This may allow us to associate the shaping and component-graph in a common framework that takes advantage of both notions, for developing connected operators on multivalued images. Beyond this simple idea, some practical issues remain to be dealt with.

After briefly summarizing recent works on component-trees, in Section 2, we define the minimal set of definitions required to make this article self-contained. To this end, Section 3 describes the notions of component-tree and component-graph in a unified graph-based formalism. Section 4 discusses the principal advantages and issues raised by coupling shaping and component-graph, for applications on multivalued images. Then, Section 5 proposes some algorithmic solutions to handle node selection and the two steps of reconstruction. An illustration in the field of 3D medical imaging is proposed in Section 6, in order to show the potentiality of this approach. A discussion concludes this article in Section 7, by emphasising the various ways to develop this framework, by extension to other kinds of hierarchies or to richer attribute spaces.

\section{Related Work on Component-Trees}

The component-tree is a compact, information lossless, hierarchical model for greylevel images. Indeed, it can represent an image in a mixed spatial / spectral space where basic operations can be interpreted in terms of image processing. In particular, filtering and segmentation $[9,14,20]$ can easily be carried out by simply selecting nodes, leading to connected operators. The versatility of the component-tree structure also has led to many other image applications, such as retrieval [21], classification [22], visualisation [23], or document binarisation [24]. 
The efficiency of the component-tree first relies on its low computation cost. In this context, many efforts were conducted to build component-trees in quasi-linear time, in sequential $[9,25]$ and distributed ways [26]. (The reader is refered to [27] for a recent survey.) The success of component-trees also relies on the development of efficient algorithmic processes for node selection. To cope with filtering and segmentation issues, two main approaches were developed. The first consists of minimizing an energy globally defined over the tree nodes, leading to define an optimal cut [28], that can be interpreted as a segmentation of the underlying image. This approach is the basis for carrying interactive segmentation [29]. The second consists of determining locally the nodes that should be preserved or discarded, based on attribute values [30]. This approach is formalized as an anti-extensive filtering framework $[9,14]$ - recalled in Section 4 - that constitutes the methodological basis of the present work.

The two main limitations of the component-tree are (1) structural: it is heavily constrained by the topological structure of the image; and (2) spectral: it is limited to greylevel (i.e., totally ordered) value images. Structural extensions of the component-tree have been proposed in [31] to deal with ordered families of connectivities, leading to component-hypertrees, and in [32] to handle images defined as valued directed graphs, leading to directed acyclic graphs (DAGs) structured over a tree. Spectral extensions were first considered by exploring marginal approaches for colour image handling [33]. Then, actual extensions of component-trees to partially-ordered value images were pioneered in [34] and further formalized in [13]. Except in specific cases where the values are themselves hierarchically organized [12], the induced data-structure, namely a component-graph, is no longer a tree, but a DAG. The antiextensive framework proposed for component-tree filtering remains valid in theory, but algorithmic issues have to be dealt with both for node selection and image reconstruction $[15,16]$.

\section{Background Notions}

We now recall some basic notions on graphs. They will allow us to describe the component-trees and component-graphs in a simple and unified formalism, and to discuss, in Sections 4 and 5, how to carry out shaping on component-graphs to handle multivalued images.

\subsection{Vertex-Valued Graphs}

A graph $\mathcal{G}_{\mathcal{L}}$ is a couple $(\Gamma, \frown)$ where $\Gamma$ is a nonempty finite set, and $\frown$ is a binary relation on $\Gamma$. The elements of $\Gamma$ are called vertices. If two vertices $x, y$ of $\Gamma$ satisfy $x \frown y$, we say that they are adjacent; any such couple $(x, y)$ is called an edge. A subgraph $\mathcal{L}^{\prime}$ of $\mathcal{L}_{\mathcal{L}}$ is a graph $\left(\Gamma^{\prime}, \frown\right)$ such that $\Gamma^{\prime}$ is a subset of $\Gamma$, equipped with the restriction of $\frown$ to $\Gamma^{\prime}$.

We consider irreflexive graphs, i.e., we never have $x \frown x$. We also consider nondirected graphs, i.e., $x \frown y \Leftrightarrow y \frown x$; the edges $(x, y)$ and $(y, x)$ are then the same.

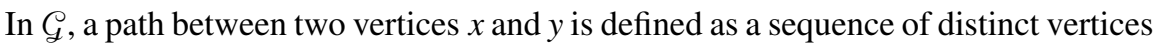
of $G_{\mathcal{L}}$ from $x$ to $y$ such that any two successive vertices are adjacent. If this path exists and is unique for any two vertices of the graph, then the graph is a tree. The connected 
components of $G_{\mathcal{L}}$ are the maximal sets of vertices that can be linked by a path. The set of all these connected components is noted $C\left[\mathcal{L}_{\mathbb{J}}\right]$; it is a partition of $\Gamma$.

Let $\mathcal{F}: \Gamma \rightarrow \mathbb{V}$ be a function such that $\mathbb{V}$ is canonically equipped with an order relation $\leq$. The triple $(\mathcal{G}, \mathbb{V}, \mathcal{F})$ is called a (vertex-)valued graph. We now define the notions of component-tree and component-graph based on this notion of valued graph.

\subsection{Component-Tree [9]}

Let $(\mathscr{G}, \mathbb{V}, \mathcal{F})$ be a valued graph. We assume that $\leq$ is a total order on $\mathbb{V}$, and that $\mathcal{L}$ is connected, i.e., $C\left[\mathcal{L}_{\mathcal{L}}\right]=\{\Gamma\}$ contains a unique connected component. Since $\Gamma$ is finite, so is the set $\mathcal{F}(\Gamma)=\{\mathcal{F}(x) \mid x \in \Gamma\} \subseteq \mathbb{V}$. Without loss of generality, we can assume that $\mathbb{V}=\mathcal{F}(\Gamma)$ and is then finite. In particular, $(\mathbb{V}, \leq)$ admits a minimum, noted $\perp$.

For any $v \in \mathbb{V}$, we define the threshold set $\Gamma_{v}=\{x \in \Gamma \mid v \leq \mathcal{F}(x)\}$. Any such threshold set induces a subgraph $\mathcal{L}_{v}=\left(\Gamma_{v}, \frown\right)$ of $\mathcal{L}_{\mathcal{L}}$. For any $v, v^{\prime} \in \mathbb{V}$ we have $v \leq$ $v^{\prime} \Leftrightarrow \Gamma_{v^{\prime}} \subseteq \Gamma_{\nu}$. In addition, for any connected component $X_{v^{\prime}}$ of $C\left[\mathcal{G}_{v^{\prime}}\right]$, there exists a (unique) connected component $X_{v}$ of $C\left[\mathcal{L}_{v}\right]$ such that $X_{v^{\prime}} \subseteq X_{v}$.

The component-tree of $(\mathcal{L}, \mathbb{V}, \mathcal{F})$, noted $\mathcal{C T}$, is the Hasse diagram of the partially ordered set $(\Psi, \subseteq)$, where $\Psi=\bigcup_{v \in \mathbb{V}} C\left[\mathcal{G}_{v}\right]$ is the set of all the connected components of the subgraphs $\mathscr{L}_{\mathcal{V}}$ obtained by successive thresholdings of $\mathscr{L}_{\mathcal{L}}$.

As suggested by its denomination, the component-tree has a tree structure. Its vertices are also called nodes. Among them, the largest is the maximum for the Hasse diagram, namely the set $\Gamma$, obtained as the unique connected component of $\mathcal{L}_{\perp}=\mathcal{L}_{\perp}$; it is the root of the tree. On the opposite side, the leaves are the minimal elements of the Hasse diagram, i.e., the nodes of $\Psi$ that do not strictly include any other nodes.

For image processing purposes, each node of $\mathcal{C T}$ generally stores a value: either an energy (for global optimization) or an attribute (for local selection); this value is most often real. In both cases, this valuation is modeled by a function $\mathcal{V}: \Psi \rightarrow \mathbb{R}$. In other words, such enriched component-tree can be interpreted as a valued graph $(\mathcal{C T}, \mathbb{R}, \mathcal{V})$.

\subsection{Component-Graph [13]}

Let $(\mathcal{L}, \mathbb{V}, \mathcal{F})$ be a valued graph. We still assume that $\mathbb{V}=\mathcal{F}(\Gamma)$ is finite and that $(\mathbb{V}, \leq)$ admits a minimum, noted $\perp$. The graph $\mathcal{L}_{\mathcal{L}}$ also remains connected, but we no longer assume that $\leq$ is a total order on $\mathbb{V}$.

We extend the notion of connected component in the following way: for any $X \in$ $C\left[\mathcal{G}_{v}\right]$, the couple $K=(X, v)$ is called a valued connected component. We note $\Theta=$ $\bigcup_{v \in \mathbb{V}} C\left[\mathcal{L}_{v}\right] \times\{v\}$ the set of all valued connected components of $\mathcal{G}_{\mathcal{L}}$, with respect to its successive thresholds. From the order relation $\leq$ and the inclusion relation $\subseteq$, we define ${ }^{1}$ the order relation $\unlhd$ on $\Theta$ as $\left(X_{1}, v_{1}\right) \unlhd\left(X_{2}, v_{2}\right) \Leftrightarrow\left(X_{1} \subset X_{2}\right) \vee\left(X_{1}=X_{2} \wedge v_{2} \leq v_{1}\right)$, which intuitively mixes the inclusion and value orders in a lexicographic way.

\footnotetext{
${ }^{1}$ Practically, when $\leq$ is a total order, the component-graph and the component-tree are isomorphic. Consequently, it would make sense to also consider the valued connected components and the order $\unlhd$ for building the component-tree, as the threshold value that leads to the generation of a connected component is useful for image modeling and reconstruction, see Equation (1).
} 


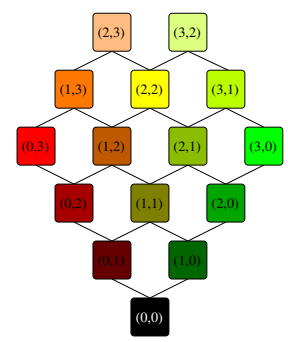

(a)

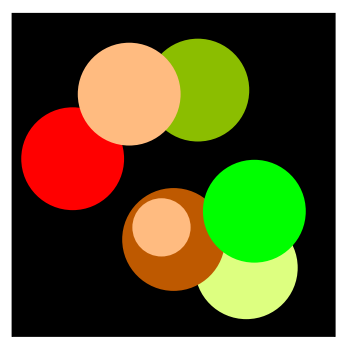

(b)

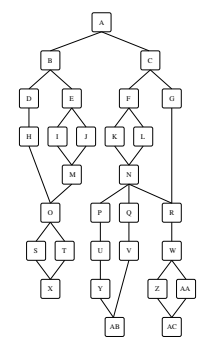

(c)

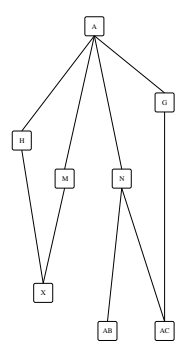

(d)
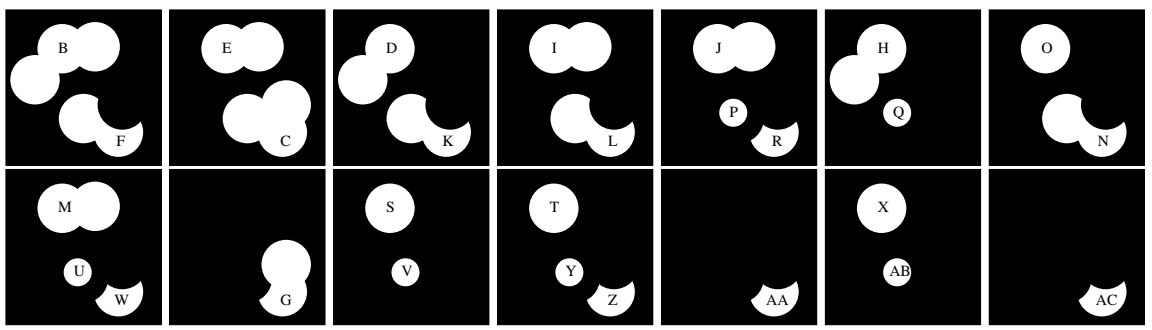

Fig. 1. (a) The Hasse diagram of the ordered set $(\mathbb{V}, \leq)$, where $\mathbb{V} \subset \mathbb{N}^{2}$ is equipped with the canonical order relation $\leq$. For the sake of readability, each value of $\mathbb{V}$ is associated to an arbitrary colour. (b) A multivalued image, viewed as a valued graph $(\mathscr{L}, \mathbb{V}, \mathcal{F})$ where $\mathcal{L}_{\mathcal{L}}$ is a part of $\mathbb{Z}^{2}$ equipped with the standard 4-adjacency relation. (c) The component-graph $\mathcal{C} \mathcal{G}$ associated to $(\mathscr{G}, \mathbb{V}, \mathcal{F})$. (d) A simplified version of the component-graph (see Section 5). Second and third rows: thresholded images obtained from (b). Each (valued) connected component is represented by a letter: A, B, C, etc. The same letters identify these components in (c,d).

The component-graph $C G_{\mathcal{L}}$ of the valued graph $(\mathscr{L}, \mathbb{V}, \mathcal{F})$ is the Hasse diagram of the partially ordered set $(\Theta, \unlhd)$ (Figure 1). It does not necessarily have a tree structure. This derives from the fact that two connected components can meet without inclusion. The component-graph has a largest node that is the maximum for the Hasse diagram, namely the set $\Gamma$; it is the root of the graph. Its leaves are the minimal elements of the Hasse diagram. The main difference is that several paths may exist between two nodes.

As for component-trees, each node of $C C_{\mathcal{G}}$ can contain an attribute value and this valuation can also be interpreted as a function $\mathcal{A}: \Theta \rightarrow \mathbb{R}$. Then, such enriched component-graph is also interpreted as a valued graph $(\mathcal{C}, \mathbb{R}, \mathcal{A})$.

\section{Shape-Space Analysis of Multivalued Images: Concept}

A (discrete) image is a mapping $\mathcal{I}$ from a finite spatial domain $\Omega$ to a value space $\mathbb{V}$. To develop connected operators, it is necessary to handle the structure of $\Omega$, i.e., to know the adjacency between its points, leading to a graph $\delta$. In addition, to develop morphological hierarchies such as component-trees and component-graphs, it is required to know the order $\leq$ on $\mathbb{V}$. An image is then modeled as a valued graph $(\mathcal{S}, \mathbb{V}, \mathcal{I})$. 


\subsection{Antiextensive Filtering With the Component-Tree}

The component-tree and the component-graph are image lossless models. More precisely, the mapping $\mathcal{I}$ can be fully recovered from the (de)composition formula

$$
\mathcal{I}=\bigvee_{(X, v) \in \Theta} C_{(X, v)}=\bigvee_{X \in \Psi} C_{(X, v)}
$$

where $C_{(X, v)}: \Omega \rightarrow \mathbb{V}$ is the cylinder function of support $X$ and value $v$, that maps $x \in X$ onto $v$ and $x \notin X$ onto $\perp$.

In the case of component-trees (i.e., for grey-level images, i.e., when $\leq$ is a total order), this formula leads to a well-defined image for $\Psi$, but also for any subset $\widehat{\Psi} \subseteq \Psi$. This consideration led to the proposal of an anti-extensive filtering framework $[9,14]$ that basically consists of three successive steps:

(i) construction of the component-tree $\mathrm{CT}$ associated to the image;

(ii) reduction of the component-tree by selection of nodes $\widehat{\Psi} \subseteq \Psi$; and

(iii) reconstruction of the result image $\widehat{I} \leq \mathcal{I}$ from the reduced component-tree $\widehat{\mathrm{CT}}$.

Step $(i)$ is carried out from a wide range of available component-tree construction methods, while Step (iii) is straightforward from Equation (1). The core of the process is Step (ii) that is dealt with by considering attribute values carried by each node of the component-tree, namely the valuation $\mathcal{V}: \Psi \rightarrow \mathbb{R}$, that guides the decision of preserving or discarding a node (together with pruning policies whenever $\mathcal{V}$ is not increasing, see $[9,14])$.

\subsection{Coupling Shaping and Component-Graphs}

Anti-extensive filtering with the component-graph On the one hand, we can extend the above anti-extensive filtering approach to images taking their values in any value space $\mathbb{V}$, without the assumption that $\leq$ is a total order. Instead of a component-tree, we then have to consider a component-graph. This allows us to process any image in the same framework as initially proposed in $[9,14]$. Nevertheless, it raises two difficulties: Step (ii) is now more complex, as the standard pruning policies have to be adapted for dealing with non-linear bottom-up or top-down node parsing; and Step (iii) can be an ill-posed problem, depending on the nature of the order $\leq$ and the preserved nodes $\widehat{\Theta}$.

Anti-extensive filtering in the shape-space [17] On the other hand, the paradigm of shaping proposes to perform anti-extensive filtering based on a double layer of component-trees, i.e., on the component-tree of the component-tree of the image. The inner component-tree is seen as an image whose points are the nodes, and grey-level values are the attributes. It is then possible to process any grey-level image in the framework initially proposed in $[9,14]$, by performing node selection in a data-structure that is no longer defined at the image level, but at a higher semantic level. This allows us also to define increasing attribute values on the outer component-tree, and to perform real-time, threshold-based node selection. The main limitation of this framework is that it considers a tree as intermediate data-structure, thus limiting its use to grey-level images. 
From "a tree on a tree" to "a tree on a graph" The formalism of valued graphs sheds light on the common structure of images, component-trees and component-graphs. As a side effect, it emphasises the fact that the inner layer of shape-space filtering only requires a graph, and not necessarily a tree. The cornerstone of this work is to consider that the initial shaping paradigm of a "tree on a tree", can be generalised to a "tree on a graph". This simple idea, summarized by Diagram (2), allows us - in theory - to process any image via a shape-based filtering.

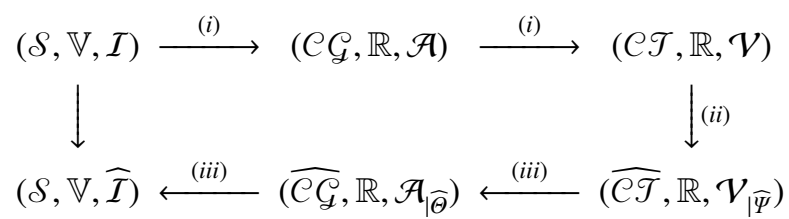

Based on the above remarks, this approach has the following virtues:

- it avoids the complex selection of nodes directly in the component-graph, since this task is indirectly carried out on the outer-layer component-tree;

- it extends the initial shaping approach beyond grey-level images;

- it inherits the good properties of shape-space filtering from increasing criteria.

Nevertheless, behind this simple idea, and its intrinsic advantages, some algorithmic issues remain to be considered, in particular for the two reconstruction steps (iii), from the component-tree to the component-graph, and then to the image. In Section 5, we propose some solutions to these issues in the case of multivalued images, that are defined as combinations of several grey-level images, opening the way to applications in multimodal / multispectral imaging.

\section{Shape-Space Analysis of Multivalued Images: Algorithmics}

The formalism of component-graphs handles valued graphs $(\mathcal{S}, \mathbb{V}, \mathcal{I})$ where $\leq$ can be any order. We focus here on the case of multivalued images where $\mathbb{V}$ is composed of $k$ spectral bands $\mathbb{V}_{i}$, each equipped with a total order. In particular, we consider the canonical partial order $\leq$ on $\mathbb{V}$ defined by $\left(v_{i}\right)_{i=1}^{k} \leq\left(w_{i}\right)_{i=1}^{k} \Leftrightarrow \forall i \in[1, k], v_{i} \leq w_{i}$.

Component-graph construction In [13], several variants of component-graphs were introduced, in particular to simplify $C C_{\mathcal{L}}$ by considering smaller subsets of $\Theta$. In the first part of Step $(i)$, that builds $C G_{\mathcal{L}}$ from $(\mathcal{S}, \mathbb{V}, \mathcal{I})$, we chose to consider the lightest version of component-graph (Figure 1(d)), i.e., the one that represents only the nodes which actually contribute to the construction of the image according to Equation (1), defined as $\ddot{\Theta}=\{(X, v) \in \Theta \mid \exists x \in X, v=\mathcal{F}(x)\}$. (For the sake of simplicity, we will now note $\ddot{\Theta}$ as $\Theta$.) This choice is motivated by the fact that such component-graphs are sufficient to process images defined in the above value space. From a complexity point of view, its construction has a lower time cost compared to the other variants of component-graphs. Moreover, its spatial complexity is in the same order as that of the initial image support $\Omega$. The component-graph $C \mathcal{G}_{\mathcal{g}}$ is built from the algorithm proposed in [15]. 
Component-graph valuation At this stage, an attribute can be associated to each node of $\Theta$, in the component-graph $C G_{2}$. We chose to consider here an attribute taking its values in $\mathbb{R}$, namely a set where all values are comparable. While alternative choices are possible (see Section 7), we assume here that a valuation $\mathcal{A}: \Theta \rightarrow \mathbb{R}$ is indeed sufficient to accurately filter the nodes, while authorising the design of a tree structure at the second layer. The criteria potentially modeled by $\mathcal{A}$ for each node $K=(X, v) \in \Theta$ can depend on: (1) spectral properties (then, we practically have $\mathcal{A}: \mathbb{V} \rightarrow \mathbb{R}$ ); (2) geometric properties (then, we practically have $\mathcal{A}: 2^{\Omega} \rightarrow \mathbb{R}$ ); (3) structural properties (then, $\mathcal{A}(K)$ depends on the relationships of $K$ within $\mathcal{C}_{\mathcal{L}}$ ); or a combination of some of these three classes. The structure of the chosen version of $C G_{\mathcal{L}}$ is relatively light, and a criterion of type (3) would be weakly relevant. For building the component-tree of the outer layer, only geometric criteria are considered here. This choice is coherent with the paradigm of shaping, and also motivated by the fact that the spectral handling of images can be carried out at the inner layer, either before or after the shaping stage.

Component-tree construction and pruning From the valued graph $(C \mathscr{G}, \mathbb{R}, \mathcal{A})$ associated to the component-graph, a shape-based component-tree can now be defined. Assuming that the relevant values of $\mathcal{A}$ are the highest, two policies can be considered to build $\mathcal{C F}$ : either as a min-tree or a max-tree. In the first case, the nodes of interest will be located near the root; in the second, they will be located near the leaves. We chose here to consider the max-tree case, that allowed us to select the relevant nodes by only preserving the distal parts, i.e., the branches of the tree. Practically, each node $Y \in \Psi$ of the component-tree $\mathcal{C T}$ is a connected component gathering nodes of a sub-

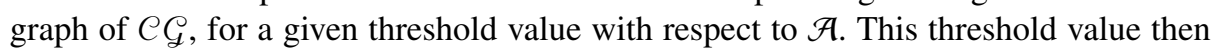
constitutes the valuation of this node, and thus defines $\mathcal{V}$. Following the above criteria classification, the valuation $\mathcal{V}$ - that is however directly obtained from a valuation of class (2) - is now a valuation of class (1) in the shape-space. In addition, it defines a monotonic (here, decreasing) criterion, allowing for an easy selection by thresholding, and avoiding the use of any specific pruning policies.

Component-graph filtering A "standard" component-tree - defined from a grey-level image - contains nodes which represent connected components of points of the image, obtained at a given threshold value. In contrast, the component-tree $\mathcal{C T}$ defined at the outer layer of the shape-space model - defined from the valued graph $\left(C G_{\mathcal{L}}, \mathbb{R}, \mathcal{A}\right)$ contains nodes that are connected components of $\Theta$ which are themselves connected components of $\Omega$. Such node $Y \in \Psi$ is then defined as a set $\left\{K_{i}=\left(X_{i}, v_{i}\right)\right\}_{i=1}^{k} \subseteq \Theta$, with $k \geq 1$. Each node $K_{i} \in Y$ is either included in another node $K_{j} \in Y$, or is a maximal element in $Y$ with respect to the $\unlhd$ relation. When dealing with geometric criteria, only these latter nodes, that contribute to define the support $\bigcup_{i=1}^{k} X_{i}$ of $Y$ in $\Omega$ are of actual interest. In other words, if $Y$ is preserved in $\widehat{\Psi}$, only these nodes should be preserved, both spatially and spectrally in the filtered image. We note $\widehat{Y} \subseteq Y$ the subset of $Y$ formed by such nodes. The other nodes of $Y$ are not taken into account; however, this is not a problem, as any node $K \in \Theta$ belongs to $\widehat{Y}$ for at least one $Y \in \Psi$. Consequently, it may be preserved based on the chosen geometric criterion, via this node. The main difference between the initially proposed shaping paradigm ("a tree on a tree") and the 
present one ("a tree on a graph"), is that the first defines any $\widehat{Y}$ as a singleton set $\{K\}$, while the second can now associate several - overlapping - nodes of $\Theta$ into a same $\widehat{Y}$, since some values of $\mathbb{V}$ may be non-comparable.

Image filtering As we deal with multivalued images, the space $(\mathbb{V}, \leq)$ is structured as a lattice. Based on this hypothesis, two strategies can be used to reconstruct the filtered image $\widehat{\mathcal{I}}$. The first - that considers each band of $\mathbb{V}$ with a same degree of relevance consists of assigning the value $v$-defined as the infimum of all the $v_{i}$ - to the reduced node $\widehat{Y}$, associated to each node $Y \in \widehat{\Psi}$ and thus to each node $K_{i}=\left(X_{i}, v_{i}\right) \in \widehat{Y}$. This policy is justified by the fact that the node $Y$ has been preserved with respect to a geometrical attribute computed for the union of all the supports $X_{i}$ of the $K_{i}$; in such conditions, the least common threshold value associated to all these nodes should be considered. However, a given node $K \in \Theta$ may belong to $\widehat{Y}_{j}$, for several nodes $Y_{j} \in \widehat{\Psi}$. In that case, the value assigned to $K$ should be defined as the supremum of all these values. This policy is justified by the fact that a node $Y \in \Psi$, defined as the union of several nodes of $\Theta$, should not lose its geometry in the filtered image. The reconstruction of the filtered image can then be formalized as follows.

$$
\widehat{\mathcal{I}}=\bigvee_{Y \in \widehat{\Psi}} C_{\left(\bigcup_{(X, v) \in \widehat{Y}} X, \wedge_{(X, v) \in \widehat{Y}} v\right)}
$$

The second strategy - that gives priority to one or several given band(s) versus others - consists of applying the first strategy on a strict subspace of $\mathbb{V}$ that corresponds to specific spectral bands. In the case where only one band is considered, the reconstructed image is a grey-level one, and the supremum and infimum on $\mathbb{V}$ considered above are simply replaced by the maximum and minimum in the considered band.

\section{Application Example: PET / CT Image Filtering}

Component-trees have been involved in the development of various tools devoted to process $3 \mathrm{D}$ medical images where the structures of interest have locally extremal values. This is the case for angiographic imaging [35,36] where high signal corresponds to flowing blood, and nuclear imaging [19] where it corresponds to high metabolic (often tumoral) activity. We illustrate the potential usefulness of our framework in this latter application field, by filtering coupled Positron Emission Tomography (PET) and Xray Computed Tomography (CT) images. These experiments remain to be clinically confirmed. As a consequence, they only constitute an illustrative proof of concept for our framework, and not an actual medical image analysis tool.

PET images (Figure 2(a)) showing metabolic activity, are classically associated to morphological CT images (Figure 2(b)) for visualizing the anatomy. Such coupled images provide complementary information. It is pertinent to process them as a unique bivalued image in order to more accurately extract the lesions and their activity.

In contrast to PET images, where the canonical order $\leq$ on $\mathbb{R}$ captures the semantics of metabolic activity, this order is - partially - meaningless with respect to the Hounsfield scale in CT. Consequently, we apply a non-injective mapping on CT images, in order to associate the lowest values to tissues of extremal (low, e.g., water and 


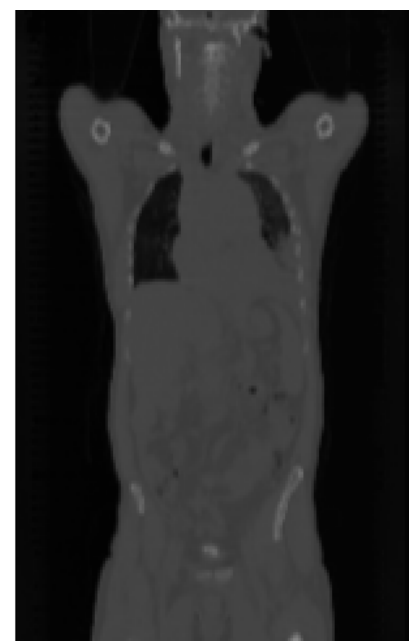

(a)

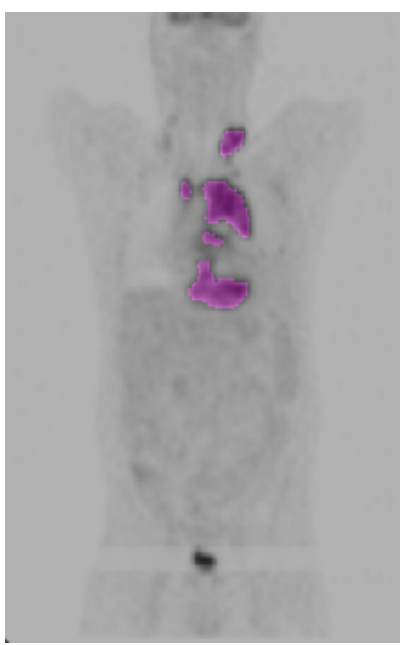

(b)

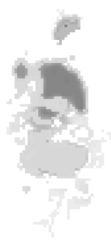

(c)

Fig. 2. Coupled CT (a) and PET (b) images. (b) Ground-truth of the lesions, in purple. (c) Multivalued shape-based filtering from $(a+b)$, visualized in the PET value space.

blood; and high, e.g., bones) intensities. The order $\leq$ on $\mathbb{R}$ for the resulting image associates the least values in the CT data to tissues which are more likely to induce false positives in PET.

The value space is subsampled to 256 values for both PET and CT, leading to a space $\mathbb{V}$ of 65536 distinct values. The criterion considered for filtering is the compactness factor [19] defined as the ratio between the extremal eigenvalues of the matrix of inertia. The filtered image is reconstructed in $\mathbb{V}$, following the first proposed policy, see Equation (3). Results of the process are exemplified in Figure 2(c), emphasising a good discrimination of lesions versus false positives, and a satisfactory spatial accuracy.

\section{Conclusion}

By coupling the two recently introduced notions of shaping and component-graph, this work opens the way to the development of new connected operators based on morphological hierarchies, and devoted to process images taking their values in rich spaces. The conceptual and algorithmic results presented here for handling multivalued images, constitute a first step toward such developments.

This preliminary study dealt with scalar attributes on the component-graph. Considering vectorial attributes [37] may enrich the potential of this framework, with the counterpart of having to cope with a component-graph at the outer layer, leading to "a graph on a tree" for grey-level imaging, and "a graph on a graph" in the most general cases.

Another limitation was to consider images where each value band was equipped with a "natural" or "semantic" order. A way to partially relax this constraint could be to 
merge component-trees and trees of shapes, to use the optimal data-structure according to the putative availability of an order for each value subspace. Recent extensions of the trees of shapes to multivalued images [11] - actually connected to the component-graph paradigm - may constitute a sound basis for such an approach.

\section{Acknowledgements}

The authors thank M. Meignan (Hôpitaux Universitaires Henri-Mondor, Lymphoma Academic Research Organisation, Créteil, France), for providing the PET / CT images.

\section{References}

1. Salembier, P., Serra, J.: Flat zones filtering, connected operators, and filters by reconstruction. IEEE Transactions on Image Processing 4(8) (1995) 1153-1160

2. Salembier, P., Wilkinson, M.H.F.: Connected operators: A review of region-based morphological image processing techniques. IEEE Signal Processing Magazine 26(6) (2009) 136157

3. Heijmans, H.J.A.M.: Theoretical aspects of gray-level morphology. IEEE Transactions on Pattern Analysis and Machine Intelligence 13(6) (1991) 568-582

4. Aptoula, E., Lefèvre, S.: A comparative study on multivariate mathematical morphology. Pattern Recognition 40(11) (2007) 2914-2929

5. Najman, L., Schmitt, M.: Geodesic saliency of watershed contours and hierarchical segmentation. IEEE Transactions on Pattern Analysis and Machine Intelligence 18(12) (1996) $1163-1173$

6. Salembier, P., Garrido, L.: Binary partition tree as an efficient representation for image processing, segmentation, and information retrieval. IEEE Transactions on Image Processing 9(4) (2000) 561-576

7. Soille, P.: Constrained connectivity for hierarchical image decomposition and simplification. IEEE Transactions on Pattern Analysis and Machine Intelligence 30(7) (2008) 1132-1145

8. Perret, B., Lefèvre, S., Collet, C., Slezak, É.: Hyperconnections and hierarchical representations for grayscale and multiband image processing. IEEE Transactions on Image Processing 21(1) (2012) 14-27

9. Salembier, P., Oliveras, A., Garrido, L.: Antiextensive connected operators for image and sequence processing. IEEE Transactions on Image Processing 7(4) (1998) 555-570

10. Monasse, P., Guichard, F.: Scale-space from a level lines tree. Journal of Visual Communication and Image Representation 11(2) (2000) 224-236

11. Carlinet, E., Géraud, T.: A morphological tree of shapes for color images. In: ICPR, Proc. (2014) 1132-1137

12. Kurtz, C., Naegel, B., Passat, N.: Connected filtering based on multivalued component-trees. IEEE Transactions on Image Processing 23(12) (2014) 5152-5164

13. Passat, N., Naegel, B.: Component-trees and multivalued images: Structural properties. Journal of Mathematical Imaging and Vision 49(1) (2014) 37-50

14. Jones, R.: Connected filtering and segmentation using component trees. Computer Vision and Image Understanding 75(3) (1999) 215-228

15. Naegel, B., Passat, N.: Colour image filtering with component-graphs. In: ICPR, Proc. (2014) 1621-1626

16. Naegel, B., Passat, N.: Toward connected filtering based on component-graphs. In: ISMM, Proc. Volume 7883 of Lecture Notes in Computer Science., Springer (2013) 350-361 
17. Xu, Y., Géraud, T., Najman, L.: Morphological filtering in shape spaces: Applications using tree-based image representations. In: ICPR, Proc. (2012) 485-488

18. Xu, Y., Géraud, T., Najman, L.: Two applications of shape-based morphology: Blood vessels segmentation and a generalization of constrained connectivity. In: ISMM, Proc. Volume 7883 of Lecture Notes in Computer Science., Springer (2013) 390-401

19. Grossiord, É., Talbot, H., Passat, N., Meignan, M., Tervé, P., Najman, L.: Hierarchies and shape-space for PET image segmentation. In: ISBI, Proc. (2015, to appear)

20. Ouzounis, G.K., Wilkinson, M.H.F.: Mask-based second-generation connectivity and attribute filters. IEEE Transactions on Pattern Analysis and Machine Intelligence 29(6) (2007) 990-1004

21. Alajlan, N., Kamel, M.S., Freeman, G.H.: Geometry-based image retrieval in binary image databases. IEEE Transactions on Pattern Analysis and Machine Intelligence 30(6) (2008) $1003-1013$

22. Urbach, E.R., Roerdink, J.B.T.M., Wilkinson, M.H.F.: Connected shape-size pattern spectra for rotation and scale-invariant classification of gray-scale images. IEEE Transactions on Pattern Analysis and Machine Intelligence 29(2) (2007) 272-285

23. Westenberg, M.A., Roerdink, J.B.T.M., Wilkinson, M.H.F.: Volumetric attribute filtering and interactive visualization using the max-tree representation. IEEE Transactions on Image Processing 16(12) (2007) 2943-2952

24. Naegel, B., Wendling, L.: A document binarization method based on connected operators. Pattern Recognition Letters 31(11) (2010) 1251-1259

25. Najman, L., Couprie, M.: Building the component tree in quasi-linear time. IEEE Transactions on Image Processing 15(11) (2006) 3531-3539

26. Wilkinson, M.H.F., Gao, H., Hesselink, W.H., Jonker, J.E., Meijster, A.: Concurrent computation of attribute filters on shared memory parallel machines. IEEE Transactions on Pattern Analysis and Machine Intelligence 30(10) (2008) 1800-1813

27. Carlinet, E., Géraud, T.: A comparative review of component tree computation algorithms. IEEE Transactions on Image Processing 23(9) (2014) 3885-3895

28. Guigues, L., Cocquerez, J.P., Le Men, H.: Scale-sets image analysis. International Journal of Computer Vision 68(3) (2006) 289-317

29. Passat, N., Naegel, B., Rousseau, F., Koob, M., Dietemann, J.L.: Interactive segmentation based on component-trees. Pattern Recognition 44(10-11) (2011) 2539-2554

30. Breen, E.J., Jones, R.: Attribute openings, thinnings, and granulometries. Computer Vision and Image Understanding 64(3) (1996) 377-389

31. Passat, N., Naegel, B.: Component-hypertrees for image segmentation. In: ISMM, Proc. Volume 6671 of Lecture Notes in Computer Science., Springer (2011) 284-295

32. Perret, B., Cousty, J., Tankyevych, O., Talbot, H., Passat, N.: Directed connected operators: Asymmetric hierarchies for image filtering and segmentation. IEEE Transactions on Pattern Analysis and Machine Intelligence (DOI:10.1109/TPAMI.2014.2366145).

33. Naegel, B., Passat, N.: Component-trees and multivalued images: A comparative study. In: ISMM, Proc. Volume 5720 of Lecture Notes in Computer Science., Springer (2009) 261-171

34. Passat, N., Naegel, B.: An extension of component-trees to partial orders. In: ICIP, Proc. (2009) 3981-3984

35. Wilkinson, M.H.F., Westenberg, M.A.: Shape preserving filament enhancement filtering. In: MICCAI, Proc. Volume 2208 of Lecture Notes in Computer Science., Springer (2001) 770-777

36. Dufour, A., Tankyevych, O., Naegel, B., Talbot, H., Ronse, C., Baruthio, J., Dokládal, P., Passat, N.: Filtering and segmentation of 3D angiographic data: Advances based on mathematical morphology. Medical Image Analysis 17(2) (2013) 147-164

37. Urbach, E.R., Boersma, N.J., Wilkinson, M.H.F.: Vector attribute filters. In: ISMM, Proc. Volume 30 of Computational Imaging and Vision., Springer (2005) 95-104 